\title{
The onset of low Prandtl number thermal convection in thin spherical shells
}

\author{
Ferran Garcia, Frank R. N. Chambers and Anna L. Watts \\ Anton Pannekoek Institute for Astronomy, University of Amsterdam, \\ P.O. Box 94249, 1090 GE, Amsterdam, the Nederlands \\ email: $\{$ f.garciagonzalez, frnchambers, a.l.watts\}@uva.nl
}

\begin{abstract}
In this study the onset of stress-free Boussinesq thermal convection in rotating spherical shells with aspect ratio $\eta=r_{\text {inner }} / r_{\text {outer }}=0.9$, Prandtl numbers $\operatorname{Pr} \in\left[10^{-4}, 10^{-1}\right]$, and Taylor numbers Ta $\in\left[10^{4}, 10^{12}\right]$ is considered. We focus on the form of the convective cell pattern that develops, and on its time scales, since this may have observational consequences for thermonuclear burning and the development of burst oscillations in the exploding oceans of accreting neutron stars (Watts (2012)).
\end{abstract}

Keywords. convection, instabilities.

\section{Introduction}

Compressible convection can develop during thermonuclear flashes in the accreted oceans of white dwarfs and neutron stars, as well as in main sequence stars and Asymptotic Giant Branch stars. These convective regions may be formed by very thin $\left(r_{\text {inner }}>\right.$ $0.8 r_{\text {outer }}$ ) layers of Helium or Hydrogen which are subject to the influence of strong temperature gradients and rotation. From nuclear physics theory the physical properties, such as kinematic viscosity or thermal conductivity, can be estimated and may give rise to very low Prandtl and large Taylor numbers. This parameter regime, in combination with very thin spherical shells, makes the study of convection extremely challenging, even in the incompressible case (Boussinesq). The latter, is the focus of this poster based on Garcia, Chambers \& Watts (2017).

\section{The model}

A spherical shell rotating about its axis of symmetry with uniform angular velocity, subject to radial gravity and to a temperature gradient, is considered. We use the same non-dimensional Boussinesq formulation of the Navier-Stokes problem as in Chandrasekhar (1981). The system is $\mathcal{S O}(2) \times \mathcal{Z}_{2}$-equivariant and the parameters are the Rayleigh number Ra, the Prandtl number Pr, the Taylor number Ta, and the radius ratio $\eta$ (see Garcia, Sánchez \& Net (2008)). Stress-free perfect thermally conducting boundaries are imposed.

The study of the onset of convection (see Garcia, Sánchez \& Net (2008)) requires finding the critical parameters (Rayleigh number, $\mathrm{Ra}_{\mathrm{c}}$, drifting frequency, $\omega_{c}$, and the azimuthal wave number $m_{c}$ ) and this reduces to find leading eigenvalues by means of an iterative (Arnoldi) method.

\section{Results}

By varying Pr and Ta the critical parameters are obtained and the transitions among spiralling columnar (SC), equatorially attached (EA) and equatorially antisymmetric/ symmetric polar $(\mathrm{AP} / \mathrm{SP})$ modes are computed and traced in the (Ta, $\mathrm{Pr}$ ) space (see 

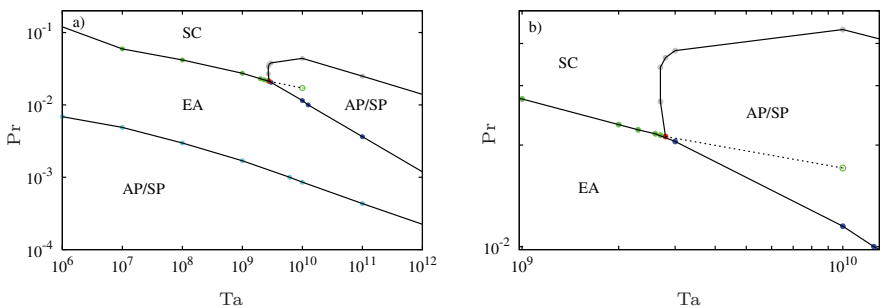

Figure 1. (a) Transitions between the different types of preferred modes. (b) Detail of (a) showing the triple point. The dashed line marks the transition between nondominant EA and SC modes.
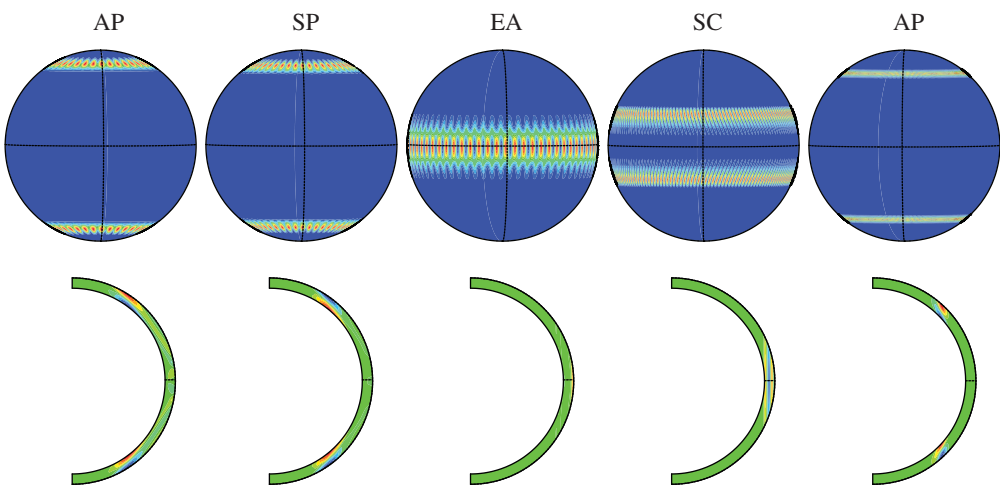

Figure 2. First row: Spherical sections of the kinetic energy density $\mathbf{v}^{2} / 2$ for the preferred modes of convection. Gray scale is not quantitative: White/Dark (Red/blue online) means most/less energetic fluid, respectively. Second row: Meridional sections of the azimuthal velocity $v_{\varphi}$. White/Dark (Red/blue online) means positive/negative $v_{\varphi}$, respectively (gray (green online) is for zero). From left to right AP mode $\left(\mathrm{Pr}=10^{-3}, \mathrm{Ta}=10^{8}, m_{c}=18\right)$, SP mode $\left(\mathrm{Pr}=2 \times 10^{-3}, \mathrm{Ta}=10^{8}, m_{c}=23,\right)$, EA mode $\left(\mathrm{Pr}=10^{-2}, \mathrm{Ta}=10^{8}, m_{c}=33\right)$, SP mode $\left(\mathrm{Pr}=10^{-1}, \mathrm{Ta}=10^{8}, m_{c}=78\right)$, and AP mode $\left(\mathrm{Pr}=3 \times 10^{-2}, \mathrm{Ta}=10^{10}, m_{c}=156\right)$.

Fig. 1 and Fig. 2). At the lowest Pr values, polar modes become the only ones that are preferred and $\mathrm{Ra}_{\mathrm{c}}$ becomes nearly constant suggesting the zero-Prandtl-limit is not far. The transition between $\mathrm{AP} / \mathrm{SP}$ and EA modes takes place at $\operatorname{Pr}_{\mathrm{P} / \mathrm{EA}}=0.68 \mathrm{Ta}^{-0.29}$ and the EA modes are superseded by $\mathrm{SC}$ at $\operatorname{Pr}_{\mathrm{EA} / \mathrm{SC}}=1.67 \mathrm{Ta}^{-0.2}$ but only for $\mathrm{Ta}<2.8 \times 10^{9}$. At larger Ta and moderate Pr we have found two additional transitions not previously described. One is between EA and AP/SP modes and the other is between AP/SP and $\mathrm{SC}$ modes taking place at larger Pr. The two transition curves intercept at $\left(\mathrm{Ta}_{3}, \mathrm{Pr}_{3}\right)=$ $\left(2.8 \times 10^{9}, 2.12 \times 0^{-2}\right)$ giving rise to a triple-point bifurcation (the dark gray point (red online) of Fig. 1) where the $\mathrm{AP} / \mathrm{SP}, \mathrm{EA}$ and $\mathrm{SC}$ modes are dominant and have very different $\left|\omega_{c}\right|$ and $m_{c}$. When nonlinearities are included, a rich variety of chaotic dynamics, characterised by different physical mechanisms, may be expected almost at the onset.

\section{Acknowledgement}

\section{ERC Starting Grant No. 639217 CSINEUTRONSTAR (PI Watts)}

\section{References}

Watts, A. L. 2012, ARAA, 50, 609

Chandrasekhar, S. 1981, in: International Series of Monographs on Physics, Oxford: Clarendon.

Garcia, F., Sánchez, J., \& Net, M. 2008, Phys. Rev. Lett., 101, 194501

Garcia, F., Chambers, F. R. N., \& Watts, A. L. 2017, Phys. Rev. Fluids, Submitted. 